\title{
When variants are reclassified-the importance of personalized communication
}

\author{
Thinking the e-mail was a system error, she almost didn't learn that her genetic test result had \\ been revised. With the advent of commercial genomic screening, who is ethically responsible \\ for communicating variant reclassification?
}

W hen I received an e-mail notifying me that the report for my genetic test was "ready," I was surprised; I had taken the test three years ago and tested positive for a $B R C A 1$ gene mutation. This puts me at a significantly increased risk of developing breast and ovarian cancer compared to the general population. My first thought, upon reading the e-mail, was that it was a system error. Out of curiosity, I logged into my account. After clicking through multiple links, I realized that the e-mail was not a glitch but rather a reclassification notice-my mutation had been upgraded from "likely pathogenic" to "pathogenic."

Among the promises of genetic testing is the ability to predict disease susceptibility in order to prevent morbidity and mortality. But for some patients, their initial test results might not be the final word-as new information becomes available, genetic variants may be reclassified as being more or less likely to cause disease. Reclassification is not uncommon. A 2018 study by Mersch et al found that among 1.45 million people who received genetic testing for hereditary cancer risk between 2006 and 2016, 6.4\% (2,868 of $44,777)$ of unique variants were reclassified ${ }^{1}$. While variants that were originally classified as 'pathogenic or likely pathogenic' or 'benign or likely benign' were rarely moved to a different clinical category, $7.7 \%$ of unique variants of uncertain significance were reclassified, with $8.7 \%$ being upgraded and $91.2 \%$ being downgraded ${ }^{1}$.

In my case, variant reclassification does not change my clinical care; most commercial testing companies already classify my particular variant as pathogenic and my oncologist has always treated it that way. This is not true for all patients. Sometimes, variant reclassification can have a significant impact on risk management ${ }^{1}$. Suppose, for instance, that a female patient's $B R C A 1$ variant of uncertain significance is upgraded to pathogenic. On the basis of this reclassification, a physician would likely recommend that the patient have a salpingo-oophorectomy (surgical removal of the Fallopian tubes and ovaries) between 35 and 40 years of age to reduce her risk of ovarian cancer. Although they are relatively rare, downgraded variants can also have significant clinical implications. For example, a patient whose variant was originally classified as pathogenic may have been encouraged to pursue certain screening or surgical risk-reducing strategies that would be inappropriate if the variant were downgraded. A change in variant status can also have a substantial emotional and psychological impact on patients. Regardless of whether a variant is upgraded or downgraded, post-hoc variant reclassification raises ethical concerns about re-contacting patients whose results have been amended.

Fortunately, efforts have been made to improve the communication of amended results $^{2}$. For instance, researchers have developed a provider interface that enables a patient's medical team to receive updates about variant reclassifications ${ }^{3}$. The success of this type of infrastructure, however, crucially depends on continued provider availability and vigilance. Provider turnover is a foreseeable challenge in genomic medicine and likely played a role in how I learned about my variant reclassification. According to the company that did my genetic testing, ordering providers are notified when a patient's variant is reclassified; after a certain number of days have passed, the amended result is shared directly with the patient. Because the genetic counselor who had originally overseen my testing was no longer available to contact me about my results, I learned about my amended report through an automated e-mail.

Due to the growth of genetic testing and concomitant expansion of the human variant database, managing individual patients' genetic test results will be an ongoing challenge. Although the primary responsibility for informing patients should fall to the ordering providers, companies still have an ethical obligation to help ensure that patients learn about their reclassified variants. This ethical duty arises from companies' role in genomic medicine; as the source and gatekeeper of patients' amended reports, companies are responsible for sharing variant reclassifications with ordering providers who, in turn, should reach out to their patients. But inasmuch as provider turnover is a reality and some patients may not receive their amended results unless companies engage with them directly, companies should take proactive steps to inform patients about their reclassified variants. To increase the likelihood that patients discuss their results with a medical provider, companies could add another level of provider oversight, such as informing the patient's primary care physician or a relevant specialist (e.g., an oncologist) about the change. In addition, instead of issuing an automated e-mail notification, testing companies should consider having one of their own genetic counselors contact patients directly.

Even in the face of incomplete knowledge, patients will continue to make healthcare decisions based on genetic testing. Testing companies should, therefore, continue to invest in developing communication strategies that recognize the evolving nature of genetic information. Doing so will improve the chances that patients receive timely updates about variant reclassifications and continue to benefit from their genetic information.

\section{Madison K. Kilbride \\ Department of Medical Ethics and Health Policy, Perelman School of Medicine at the University of \\ Pennsylvania, Philadelphia, PA, USA. \\ e-mail:madisonk@upenn.edu}

Published online: 13 January 2020 https://doi.org/10.1038/s41591-019-0707-9

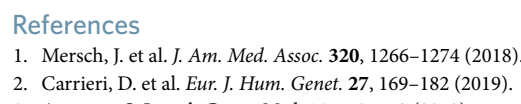

